\title{
Design and Implementation of Double Base Integer Encoder of Term Metrical to Direct Binary Code Application
}

\author{
Takialddin A. Al Smadi
}

Department of Communications and Electronics Engineering, College of Engineering, Jerash University, Jerash, Jordan. Email: dsmadi@rambler.ru

Received June $1^{\text {st }}, 2013$; revised July $1^{\text {st }}, 2013$; accepted July $10^{\text {th }}, 2013$

Copyright (C) 2013 Takialddin A. Al Smadi. This is an open access article distributed under the Creative Commons Attribution License, which permits unrestricted use, distribution, and reproduction in any medium, provided the original work is properly cited.

\begin{abstract}
The digital processing signal is one of the subdivisions of the analog digital converter interface; data transfer rate in modern telecommunications is a critical parameter. The greatest feature of parallel conversion rate (4-bit parallel Flash $5 / \mathrm{s}$ converter) is designed and modeled in 0.18 micron CMOS technology. Low speed swing operation as analog and digital circuits leads to high speed of low power operation power with $70 \mathrm{mVt} 1.8 \mathrm{~V} \mathrm{~A} / \mathrm{D}$ converter from the power dissipated during operation in the $5 \mathrm{GHz}$ range. Average offset is used to minimize the effect of the bias of a comparator. This paper contains the 8-bit encoder of the metrical term code to direct binary code decreasing power consumption, which is shown by results and comparison with other designs using computer simulation. The results of the flash ADC time-interleaved are a more significant improvement in terms of power and areas than those previously reported.
\end{abstract}

Keywords: ADC; CMOS VLSI; High Speed Data Converters; Code

\section{Introduction}

Digital communication tools with high data rate, high speed broadband, radar and optical communications, these applications require 4 to 6 bit resolution at rates of $1 \mathrm{GHz}$ or beyond.

Several papers have been published previously in the 4-bit Flash ADC [1]. The multi-GHz A/D sampling rate is achieved by using interleaved time architecture.

Because of the gain and offset of the inconsistencies among the various channels of ADC time-interleaved architecture usually requires numerical methods [2].

These calibrations scheme to significantly increase the power and/or Flash ADC area. The proposed architecture $5 / \mathrm{s}$ speed is achieved based on low swing in full operation of the ADC. Two stages on average bias resistor give relations in 3.65.

Thus no digital calibration is required, encoding to significant savings in power and scope.

Resistor ladder generates tap voltage 21 voltage references from two clean $0.9 \mathrm{~V}$ and $1.6 \mathrm{~V} .21$ multi-stage comparators, including 15 major and over-3 range comparators on each end of the array, compare the input signal voltage from the crane and generate code thermome- ter. Finally, the current encoder mode logic (CML) translates the code with binary thermometer through the intermediate gray code $[3,4]$.

No external track and hold $(\mathrm{t} / \mathrm{h})$ is used in the ADC. Instead, the sample is distributed in the first latch comparator array.

\section{Comparator Array}

Preamplifier an array is a regenerative latch that operates as a distributed monitor and keep, and two additional tabs are available to achieve further enhancement and differential swing low level in the comparators' output [5]. No hours available for preamp which gives a continuous signal to the first latch. Shows the schematic of the preamplifier to Figure 1.

\section{Related Works}

Development of Eight-Encoder Design Steps

Inputs to the capacity of the output code from four to eight digits are based encoders lower order Exam.

The XOR Gate schematic CML shows after piping the propagation delay of the slow pipeline stage limits the 
operating frequency. So to implement an efficient scheme of piping, it is desirable to have some delays at all stages [6].

Diagram of the encoder is shown in Figure 2 and developed in accordance with prudent use of a minimum number of components, which reduces the space occupied by the on-chip. MOSFETs-substrate transistors with n-channel $T_{1}, T_{4}$ and $T_{5}$ are connected to the negative rail power supply Vss, and the substrate p-MOSFETs with channel $\mathrm{T}_{2}, \mathrm{~T}_{3}$ and $\mathrm{T}_{6}$ to the positive rail $\mathrm{Vdd}$ [7].

Encoder (Figure 2) consists of two CMOS-keys on the basis of transistors $\mathrm{T}_{1}, \mathrm{~T}_{2}$ and $\mathrm{T}_{5}, \mathrm{~T}_{6}$, which are controlled by the voltage at the input $\mathrm{X}_{1} ; \mathrm{Y}_{0}$ determines the MSB output binary code. Input $\mathrm{X}_{1}$ comes from the output of the comparator switching threshold which corresponds to the middle of the two-digit range input ADC. Keys at the same switching voltage $\mathrm{X}_{0}$ and $\mathrm{X}_{2}$ from the outputs of the other two comparators to generate low-order output $\mathrm{Y}_{1}$ binary ADC. Based on the proposed scheme can be implemented three-bit encoder, where a block with twodigit designation DEC encoder according Proceeding similarly, we obtain a four-digit encoder circuit based on the three-digit encoder [8]. This requires the use of twoinput multiplexers labeled MUX, Figure 3 shows its' scheme. Substrate MOSFET with n-channel $\mathrm{T}_{2}, \mathrm{~T}_{4}, \mathrm{~T}_{5}$, $\mathrm{T}_{6}, \mathrm{~T}_{8}$ and $\mathrm{T}_{10}$ are connected to the negative rail power supply $\mathrm{V}_{\mathrm{ss}}$, and the substrate MOSFETs p-channel $\mathrm{T}_{1}, \mathrm{~T}_{3}$, $\mathrm{T}_{7}$ and $\mathrm{T}_{9}$ - to the positive rail $\mathrm{V}_{\mathrm{dd}}$. The multiplexer is a signal at the address input $\mathrm{A}$. When the signal at input $\mathrm{A}$, equal logical unit, the output signal from the input $D_{1}$, and when the signal A, equal to a logical zero, with input $\mathrm{D}_{2}$. Inverters based on transistors $\mathrm{T}_{7}-\mathrm{T}_{10}$ are the buffer elements. Thus, increasing the bit similar to Figure 3.

Modeling was conducted with (tt, ss, ff, snfp, fnsp) for three values of temperatures $-40^{\circ} \mathrm{C}$ and $27^{\circ} \mathrm{C}, 85^{\circ} \mathrm{C}$. The

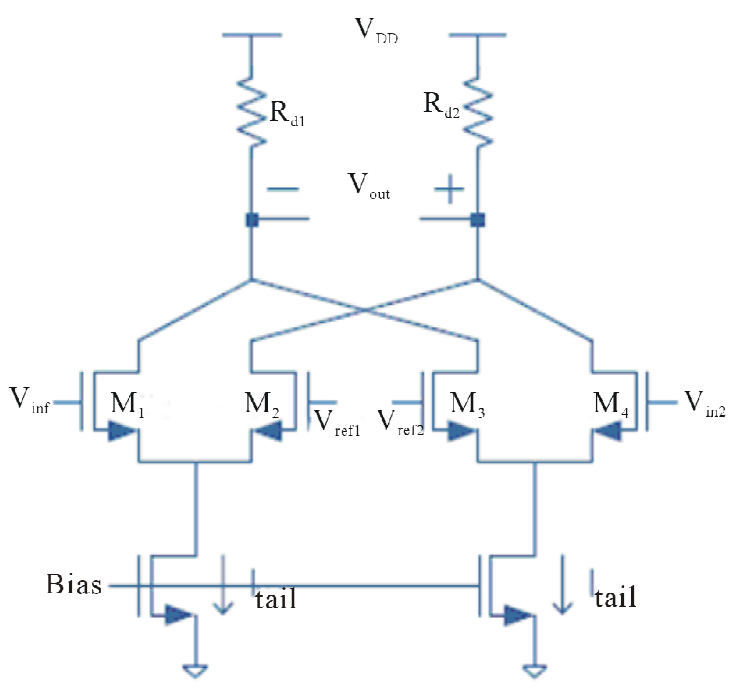

Figure 1. Schematic of the preamplifier. results are presented in Table $\mathbf{1}$

\section{Material and Methods Simulation Results}

Power characteristics of the encoder performed using MOSFETs Cadence Virtuoso based on $180 \mathrm{~nm}$ CMOS technology from UMC to $1.8 \mathrm{~V}$ single supply [9]. Delay time-shift eight-evaluated by the response of the encoder output LSB Y8 direct binary code when the input code in the thermometric all 255 bits of logic zero to logic one on the front, and vice versa trailing edge, due to the encoder circuit solution. According to the presented in the previous section schemes, the most time-delay switch will have LSB output direct binary code. Clock frequency. Winning on the power consumption of circuit solutions presented encoder compared to known analogs [8-10] can evaluate on the basis of the simulation results. It is necessary to implement the conversion of power consumption being compared encoder $\left(P_{\text {ref }}\right)$ the equivalent Encoder, executed in the same way, 8-bit word length, manufactured in $180 \mathrm{~nm}$ CMOS technology and has a clock speed of $1 \mathrm{GHz}$. In this case, the supply current from the translation were held constant. Then the change in power consumption can be estimated: at another bit by the coefficient [9].

$N_{e q}-N_{\text {ref }}$ bit Equivalent, and compares the encoder respectively, when changing technology-a factor $\frac{E_{e}}{E_{c}}$.

$E_{e q}$ - voltage encoder, made in $180 \mathrm{~nm}$ CMOS technology $\left(E_{e q}=1.8 \mathrm{~B}\right), E_{r e f}$-encoder supply voltage being compared; when the clock- $\frac{F_{e}}{F_{c}}, F_{e q} \& F_{\text {ref }}-$ clock frequency equivalent to the developed and the compared encoders respectively. Then the equivalent power consumption is defined as

$$
F_{e q}=2^{N_{e q}-N_{r e f}} p_{r e f} \frac{E_{e q}}{E_{r e f}} \frac{F_{e q}}{F_{r e f}} .
$$

Table 2 under the conditions of winning based on estimates of power consumption circuitry solutions developed encoder compared to known analogs is table.

The minimal gain in power consumption is obtained for eight-ADC encoder from Gain in power consumption is obtained for eight-ADC encoder from [10]

$$
\frac{P_{e q}}{P_{d e v}}=\frac{0.438}{0.439} \frac{1.8}{0.7} \frac{1}{2}=1.3 \mathrm{~T}
$$

$P_{t}$ - average power consumption of the developed encoder. The maximum gain in power consumption is obtained for the encoder [11] on the basis of multiplexers.

$$
\frac{P_{e q}}{P_{d e v}}=\frac{0.254}{0.449} \frac{1}{0.1}=45.3 \mathrm{~T}
$$




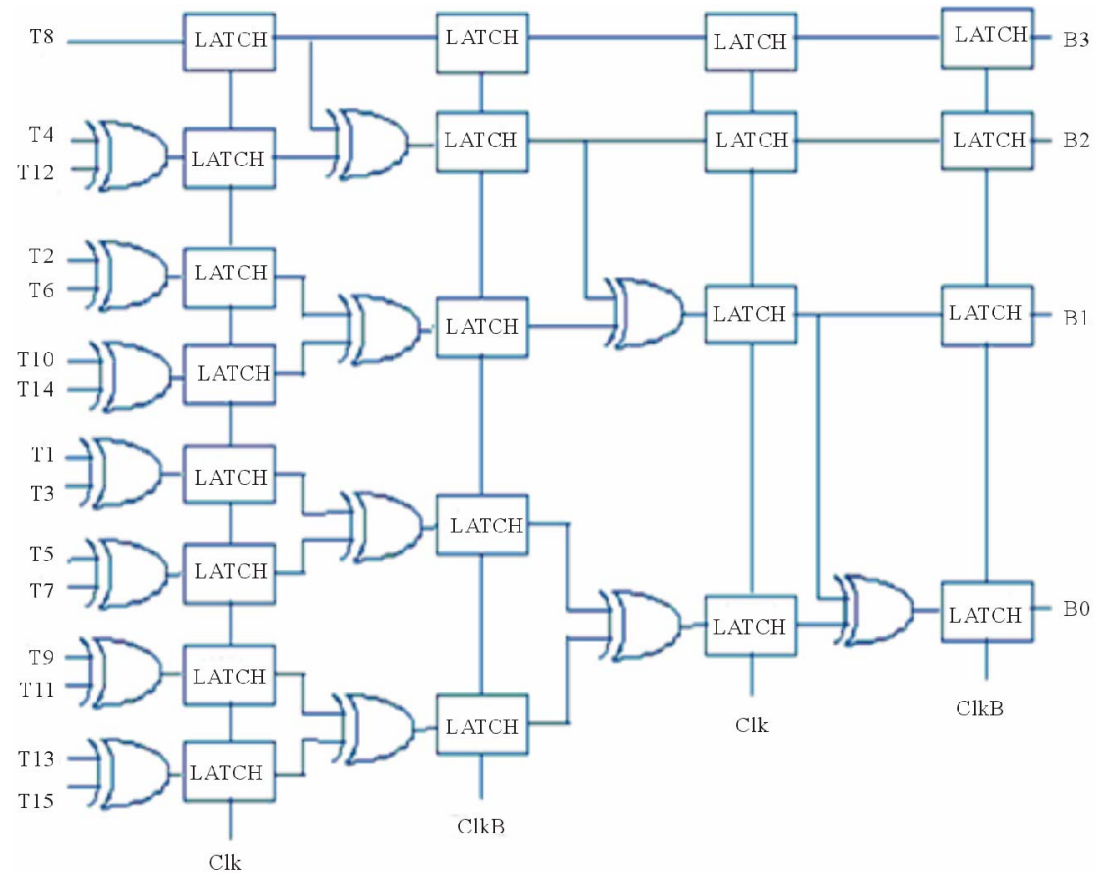

Figure 2. Implementation of encoder with four stage pipeline and only one type of gate.

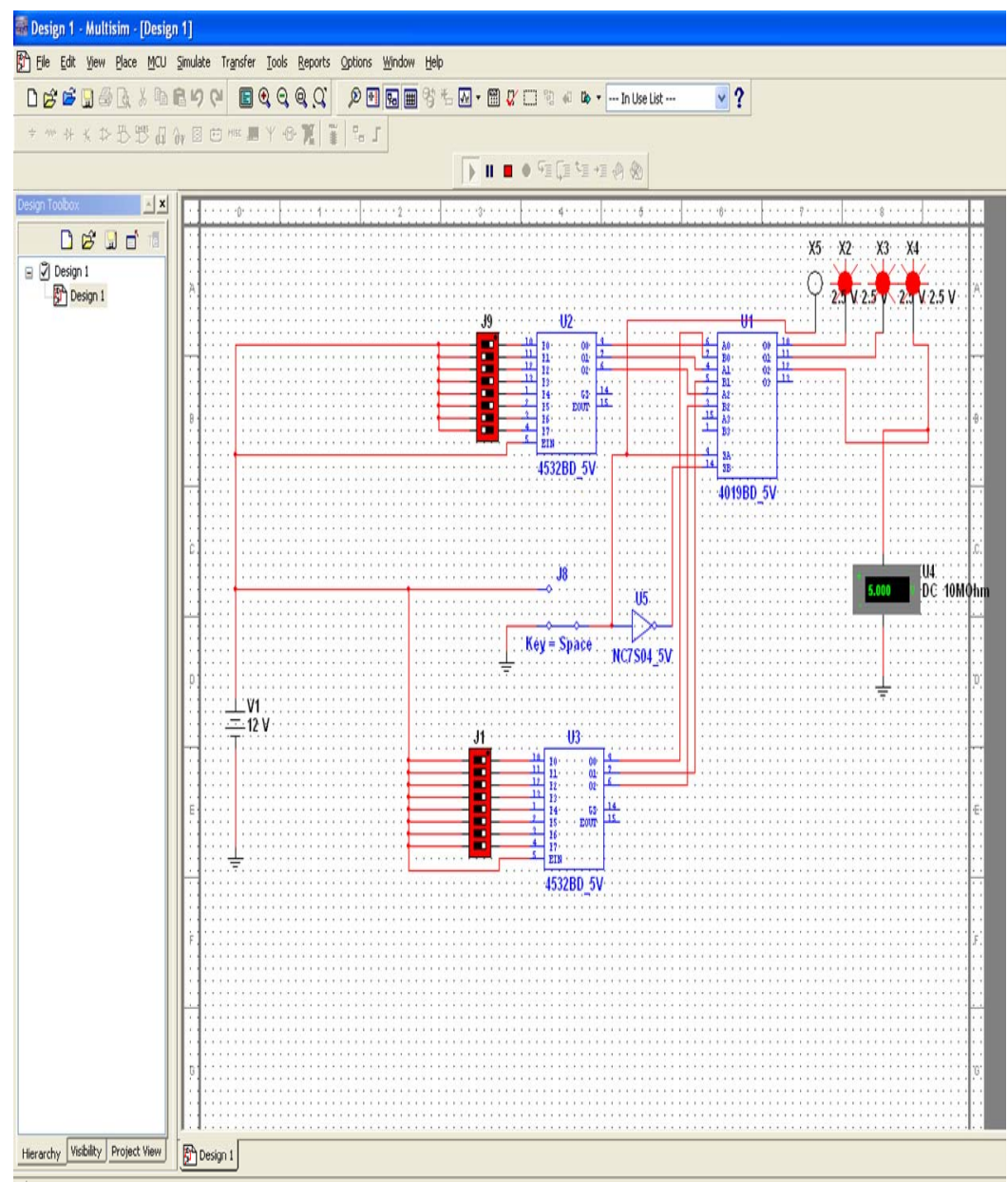

Figure 3. Eight-bit encoder. 
Table 1. Consumption of the encoder.

\begin{tabular}{|c|c|c|c|c|c|}
\hline Terms & $\mathbf{T}^{\circ} \mathbf{C}$ & Time-delay switch & Duration of the recession & Front time & Power consumption \\
\hline & -40 & 584 & 24 & 43 & 430 \\
\hline \multirow[t]{3}{*}{$t t$} & 27 & 640 & 29 & 50 & 442 \\
\hline & 85 & 689 & 32 & 55 & 461 \\
\hline & -40 & 881 & 31 & 59 & 411 \\
\hline \multirow[t]{3}{*}{ ss } & 27 & 957 & 38 & 70 & 429 \\
\hline & 85 & 1020 & 44 & 78 & 446 \\
\hline & -40 & 431 & 19 & 33 & 446 \\
\hline \multirow[t]{3}{*}{ ff } & 27 & 475 & 23 & 39 & 460 \\
\hline & 85 & 513 & 26 & 43 & 485 \\
\hline & -40 & 666 & 24 & 44 & 469 \\
\hline \multirow[t]{3}{*}{ snfp } & 27 & 726 & 29 & 51 & 483 \\
\hline & 85 & 778 & 34 & 56 & 504 \\
\hline & -40 & 434 & 24 & 45 & 408 \\
\hline \multirow[t]{2}{*}{ Snsp } & 27 & 588 & 28 & 52 & 425 \\
\hline & 85 & 634 & 23 & 58 & 443 \\
\hline Mean value & & 674 & 29 & 52 & 449 \\
\hline The maximum value & & 1020 & 44 & 78 & 504 \\
\hline
\end{tabular}

Table 2. Decreased power consumption.

\begin{tabular}{ccc}
\hline Encoder & CMOS & Power consumption. (t) \\
\hline Full Adders & & 24.7 \\
Memory Elements & 0.18 & 41.6 \\
Multiplexers & & 45.2 \\
Logic Elements & 1.4 \\
\hline
\end{tabular}

\section{Conclusion}

The paper proposed a circuit solution to thermometric encoder code in straight binary code. Eight-circuit simulation performed in CAD Cadence Virtuoso for $180 \mathrm{~nm}$ CMOS technology with a unipolar voltage $1.8 \mathrm{~V}$. Maximumly delayed time-shift is about $1 \mathrm{~ns}$, which allows the use of the scheme in the processing of signals with a frequency of $1 \mathrm{GHz}$ along with existing analogues. Average power consumption does not exceed $500 \mathrm{~mW}$. All else being equal to a gain on the power consumption in comparison, the known digital calibration can be added to implement ultra-high-speed time-interleaved ADCs to 40 times. The reduction in the number of comparators architecture makes it useful in portable ECG systems which operate at low voltage and low frequency range.

\section{REFERENCES}

[1] R. Yousry, E. Hegazi and H. F. Ragai, "A Third-Order
9-Bit 10-MHz CMOS Modulator with One Active Stage," IEEE Transactions on Circuits and Systems I: Regular Papers, Vol. 55, No. 9, 2008, pp. 2469-2482.

[2] C. Sandner, M. Clara, A. Santner, T. Hartig and F. Kuttner, "A 6-Bit 1.2-GS/s Low-Power Flash-ADC in $0.13 \mu \mathrm{m} \mathrm{Di-}$ gital CMOS," IEEE Journal of Solid-State Circuits, Vol. 40, No. 7, 2005, pp. 1499-1505.

[3] P. Nuzzo, P. Van Der Plas, G. De Bernardinis, R. Van Der Perre, L. Gyselinckx and B. Terreni, "A $10.6 \mathrm{Mw} /$ $0.8 \mathrm{Pj}$ Power-Scalable $1 \mathrm{Gs} / \mathrm{S} 4 \mathrm{~B}$ Adc in $0.18 / \mathrm{Spl} \mathrm{Mu} / \mathrm{M}$ Cmos with 5.8Ghz Erbw," The 43rd ACM/IEEE of the Design Automation Conference, San Francisco, 2006, pp. 873-878.

[4] K. Uyttenhove and M. S. J. Steyaert, "A 1.8-V 6-Bit 1.3-Ghz Flash Adc in 0.25-MM Cmos," IEEE Journal of Solid-State Circuits, Vol. 38, No. 7, 2003.

[5] M. F. Chang, "Semiconductor Technology Considerations in High Speed Data Conversion," IEEE of Compound Semiconductor Integrated Circuit Symposium, 2004, pp. 31-34.

[6] G. L. Madhumati, K. Ramakoteswara Rao and M. Madhavilatha, "Comparison of 5-Bit Thermometer-to-Binary Decoders in $1.8 \mathrm{~V}, 0.18 \mu \mathrm{m}$ CMOS Technology for Flash ADCs," 2009 International Conference on Signal Processing Systems, Singapore, 15-17 May 2009, pp. 516-520.

[7] S. Sheikhaei, S. Mirabbasi and A. Ivanov, "An Encoder for a $5 \mathrm{GS} / \mathrm{s}$ 4-Bit Flash ADC in $0.18 \mu \mathrm{m}$ CMOS," Canadian Conference of the Electrical and Computer Engineering, May 2005, pp. 698-701.

[8] T. A. Al Smadi, "Computer Application Using Low Cost 
Smart Sensor," International Journal of Computer Aided Engineering and Technology, Vol. 4, No. 6, 2012, pp. 567-579. http://dx.doi.org/10.1504/IJCAET.2012.049572

[9] Y. Lavania, G. T. Varghese and K. K. Mahapatra, “An Ultra Low Power Encoder for 5 Bit Flash ADC," International Conference on Emerging Trends in VLSI, Embedded System, Nano Electronics and Telecommunication System (ICEVENT), 2013, pp. 1-5.

[10] M. S. Nguyen and J. Kim, "Design and Implementation of Double Base Integer Encoder in the Flash ADC," The 6th International Conference on Electrical Engineering, Pattaya, 6-9 May 2009, pp. 496-499.

[11] K. Uyttenhove and M. Steyaert, "Speed-Power-Accuracy Tradeoff in High-Speed CMOS ADCs," IEEE Transactions on Circuits and Systems II: Analog and Digital Signal Processing, Vol. 49, No. 4, pp. 280-287.

http://dx.doi.org/10.1109/TCSII.2002.801191 